\title{
Comentario epidemiológico sobre el primer caso colombiano de leishmaniasis visceral
}

\author{
Alberto Morales ${ }^{1}$, Gerzaín Rodríguez ${ }^{2}$
}

\begin{abstract}
Resumen
El primer caso de leishmaniasis visceral demostrado en Colombia fue el de una niña de 37 meses de edad, fallecida en 1943, cuyo hígado, estudiado por viscerotomía, reveló la enfermedad. La paciente vivía en la vereda Chaparral de San Vicente de Chucurí, Santander, y desde entonces se consideró el caso como autóctono de este municipio. Observaciones epidemiológicas, realizadas desde 1972, 29 años después de hecho el diagnóstico, relacionadas con la ecología del vector, con el sitio de nacimiento y la procedencia inicial de la niña, permiten concluir que la paciente adquirió la infección en la vereda San Nicolás del municipio de Lebrija, Santander, área que sí es hábitat adecuado para el desarrollo de Lu.longipalpis, vector de la enfermedad.
\end{abstract}

\section{Summary}

The first case of visceral leishmaniasis demonstrated in Colombia was that of a 3 year-old girl, who died in 1943 and whose liver, in postmortem viscerotomy study, revealed the disease. The patient lived in the rural area of Chaparral in San Vicente de Chucurí, located in the Santander department. Since then, the case was considered as being autochthonous form Chucurí. Epidemiological observations made since 1972,29 years after the diagnosis was made, related to the vector's ecology, the girl's place of birth and origin, led to the conclusion that the disease was acquired in the rural area of San Nicolás in Lebrija, Santander department, which is a suitable habitat for the development of Lu. longipalpis, the vector of the disease.

El 12 de noviembre de 1943 falleció una niña de 37 meses de edad, en la finca San Jerónimo, localizada en la vereda Chaparral del municipio de San Vicente de Chucurí, Santander. El doctor Augusto Gast Galvis diagnosticó leishmaniasis visceral al examinar cortes del hígado de esta niña, obtenidos por viscerotomía (figura 1), comprobándose así el primer caso de esta entidad en Colombia.

En las conclusiones de la publicación de Gast y Rengifo (1) sobre este caso se lee: "Fue hallado en el municipio de San Vicente de Chucurí (Santander) un caso autóctono de leishmaniasis visceral". Es decir, desde su descripción original, este caso. se ha considerado autóctono de San Vicente de Chucurí. Sin embargo, el análisis de nueva evidencia epidemiológica reunida por uno de nosotros (A.M.), nos hace deducir que esta niña no adquirió su enfermedad en ese municipio. La discusión de este asunto es el objeto de esta publicación.

1 Investigador Emérito, Laboratorio de Entomología, Instituto Nacional de Salud, Apartado aéreo 80334, Santa Fé de Bogotá, Colombia, S.A.

2 Coordinador de Investigaciones, Laboratorio de Patología, Instituto Nacional de Salud; Profesor Titular de Cátedra, Departamento de Patología, Facultad de Medicina, Universidad Nacional, Santa Fé de Bogotá. 


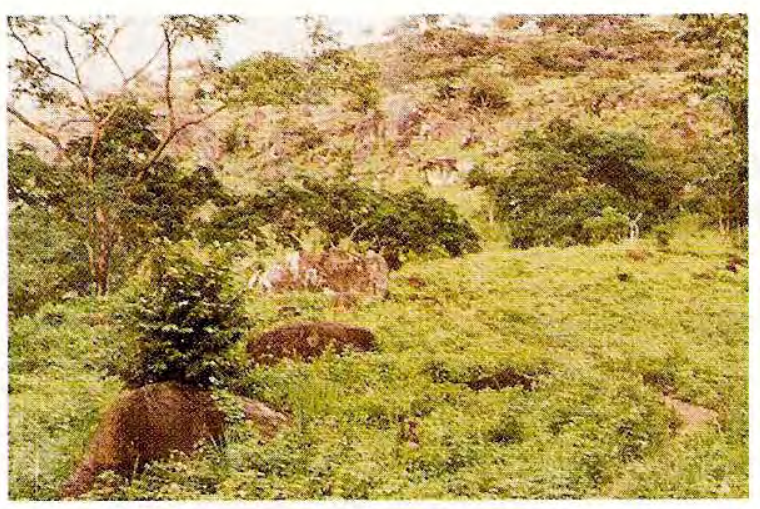

Figura 1. Vereda El Callejón, Ricaurte, Cundinamarca. Aspecto de las rocas que sobresalen en las montañas.

\section{Antecedentes}

El Laboratorio de Entomología del Instituto Nacional de Salud ha venido estudiando durante años los insectos vectores de leishmaniasis cutánea, mucocutánea y visceral. En desarrollo de estas investigaciones se decidió visitar el sitio en donde residía la niña cuando murió, con el propósito de buscar allí Lutzomyia longipalpis y de confirmar una vez más que en Colombia donde hay leishmaniasis visceral existe L. longipalpis (2), aunque Travi y colaboradores han sugerido que Lutzomyia evansi es un vector de L. chagasi (3). Esta especie no ha sido encontrada hasta ahora en San Vicente de Chucurí, Santander. Se tenía interés en observar esta área desde el punto de vista ecológico, por ser el sitio donde se había descrito el primer caso de leishmaniasis visceral en el país.

Después de consultar la publicación que describía el lugar (1), de conversar con el doctor Gast y de revisar su diario que contiene mucha de la información pertinente, nos fue fácil localizar el sitio exacto de la muerte de la paciente, al cual llegamos 29 años después en septiembre de 1972.

La publicación original dice: "La niña enfermó en la vereda Chaparral a unos $15 \mathrm{~km}$ de San Vicente de Chucurí cerca de la carretera que conduce a Bucaramanga, poco antes de llegar a la hacienda de La Soledad, unos 300 metros más abajo de la carretera en dirección al río Chucurí" (1). Efectivamente, encontramos la quebrada La Soledad en un sitio denominado Villa Elisa; por este sitio y en dirección al río
Chucurí penetra el camino que conduce a la casa donde vivía la paciente. Conversamos con un señor, vecino y conocido de la familia de la niña fallecida, quien recordaba muy bien las circunstancias de la muerte de la paciente e, inclusive, acompañó a los investigadores que en 1944 estuvieron haciendo el estudio epidemiológico del caso y aún podía mencionar los apellidos de algunos de ellos.

El lugar fue descrito así: "La habitación está rodeada de cultivos de café, cacao, yuca, caña y plátano; a unos $80 \mathrm{~m}$ hacia el norte pasa la quebrada La Soledad, afluente del río Chucurí y junto a esta vertiente hay una mancha de monte" (1). Observamos los mismos tipos de cultivos y las únicas diferencias eran que la casa, originalmente de madera y techo de paja, había sido mejorada substancialmente y no había mancha de monte, pues había sido talada. Nos llamó la atención que la formación vegetal del área, tanto en su descripción original como en la actual, corresponde a bosque húmedo tropical, según la Memoria explicativa sobre el mapa ecológico (4). La leishmaniasis visceral se presenta en Colombia en áreas xerofíticas secas o con moderada humedad y en zonas de bosque seco tropical, con suelo pedregoso y abundantes rocas. Así ha ocurrido en los casos descritos en Cundinamarca, La Guajira, Huila, Santander y Tolima (figuras 2 y 3 ).

Es en este tipo de hábitat en el que se desarrolla y prospera el vector, Lu. longipalpis y no en uno como el que veíamos en la vereda Chaparral.

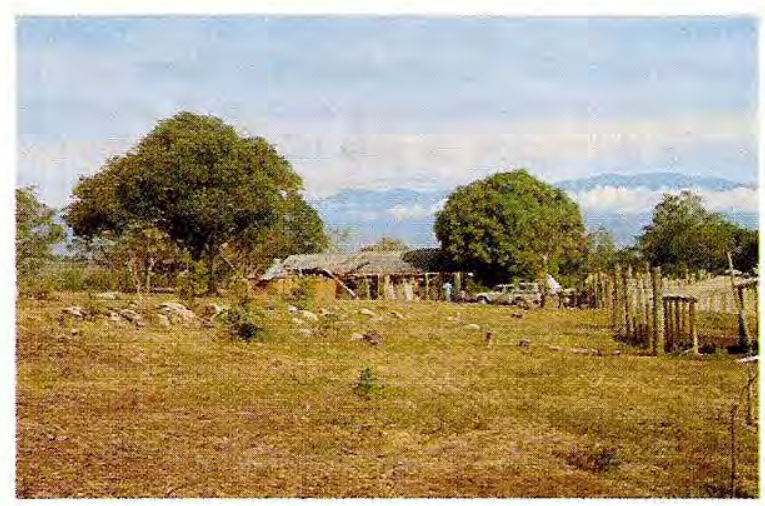

Figura 2. Vereda Nazareth, Palermo, Huila; casa con perro positivo para LVA. 


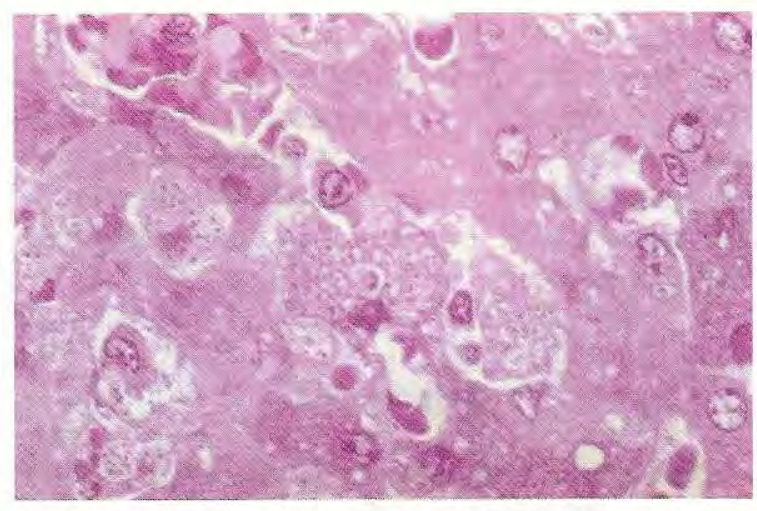

Figura 3. Células de Kupffer con abundantes amastigotes fagocitados. Caso princeps de LVA,1943; hígado HE 100X.

Durante los días 13 y 14 de septiembre de 1972, adelantamos una búsqueda cuidadosa de Lu. longipalpis intra, peri y extradomiciliaria en esta área, con resultados negativos.

En marzo de 1944 se realizaron capturas de Lutzomyia spp. cerca de la casa y en otros sitios de la vereda (1); el 16 y 17 de agosto de 1944, los doctores Augusto Gast, Ernesto Osorno y Octavio Mangabeira, hijo, capturaron ejemplares de Lutzomyia en el interior de la habitación, empleando gallinas como cebo. También recolectaron ejemplares de Lutzomyia y Brumptomyia en otros lugares del área, en huecos de árboles y en cuevas de armadillos. El material reunido fue estudiado años más tarde por el doctor Italo A. Sherlock (5), sin encontrar LU. longipalpis. Dentro de las especies determinadas por Sherlock, Lu.camposi, Lu.marajoensis, Lu.salulensis, Lu.triramula, Lu.walkeri, ninguna, hasta donde se sabe, es vectora de leishmaniasis visceral. Tampoco se encontró en este material, ni se ha demostrado después en San Vicente de Chucurí, Lu. evansi.

\section{Hipótesis}

Estas consideraciones nos hicieron suponer que la niña pudo haberse infectado en Lebrija, Santander, municipio de donde era oriunda. Por esto se decidió localizar al padre de la niña (la madre había muerto en 1966) y después de algunas averiguaciones lo encontramos en una finca de la vereda San Nicolás del municipio de Lebrija, el 25 de enero de 1973. Manifestó que la niña nació a comienzos de diciembre de
1940 en la vereda San Nicolás de Lebrija, en donde permaneció hasta su traslado a la finca San Jerónimo de San Vicente de Chucurí, en noviembre o diciembre de 1942, cuando tenía dos años de edad. Dijo que unos seis meses antes de morir, la niña presentó fiebre, pérdida del apetito, enflaquecimiento y "abultamiento de la barriga", cuando hacía seis meses que vivían en San Vicente de Chucurí.

Ese mismo 25 de enero de 1973 se buscó Lu. longipalpis en unas rocas a orillas del caño San Nicolás, en la vereda de este mismo nombre, con resultado negativo. Pero el tiempo que pudimos emplear en la búsqueda fue sólo de 30 minutos.

En nuestra opinión, el primer caso de leishmaniasis visceral en Colombia es autóctono de la vereda San Nicolás de Lebrija, Santander, por las siguientes razones:

1. La vereda Chaparral en San Vicente de Chucurí, en donde la niña residió el año anterior a su muerte, es de bosque húmedo tropical, al igual que las veredas circunvecinas. Esta ecología es impropia para el desarrollo del vector de la leishmaniasis visceral $L u$. longipalpis. Tampoco es hábitat adecuado para Lu.evansi, otro vector de $L u$. chagasi (3).

2. No se ha comunicado ningún otro caso de leishmaniasis visceral procedente de esta zona de San Vicente de Chucurí, 52 años después de la observación original.

3. En dos ocasiones se ha buscado cuidadosamente $L u$. longipalpis en la vereda Chaparral y en otros lugares vecinos de San Vicente de Chucurí, sin lograrse demostrar la existencia de esta especie.

4. La niña vivió dos años en la vereda San Nicolás de Lebrija, región de bosque seco tropical, de ecología favorable para el desarrollo de Lu. longipalpis, el vector comprobado de la leishmaniasis visceral en Colombia. En veredas y municipios vecinos a San Nicolás que tienen una ecología semejante, tales como La Girona, Palo Gordo, Girón y Piedecuesta, se han comprobado cinco casos de leishmaniasis vis- 
ceral (6-8). En la vereda Palo Gordo de Girón, de donde proceden dos de estos casos (8), hemos comprobado una alta densidad de Lu. longipalpis.

5. Aunque no encontramos Lu.longipalpis en la vereda San Nicolás de Lebrija, creemos que ello se debió al corto tiempo destinado a su búsqueda y que una investigación más prolongada lo pondría en evidencia. En el transcurso de los años no ha sido posible repetir esta búsqueda, que algún día tendrá que realizarse.

6. La duración de la enfermedad clínica de la niña fue de unos 6 meses, a juzgar por los datos suministrados por el padre, aunque en el trabajo original (1) se consigna que esta duración fue de 4 meses. La paciente vivió 12 meses en San Vicente de Chucurí, en 4-6 de los cuales tuvo signos y síntomas francos de leishmaniasis visceral, con los cuales falleció y que se ajustan a lo conocido, pues la evolución natural de esta enfermedad conduce a la muerte del 75 $95 \%$ de los pacientes en un período de 2 años (9).

El estado de la salud de la niña en los 6 meses iniciales en San Vicente de Chucurí fue aparentemente normal, según el padre, lo cual podría corresponder a un desarrollo insidioso de la enfermedad o a su período de incubación, que es variable. Puede ser tan corto como de 10 días y en la mayoría de los casos es de 2-6 meses (10). Pero, también puede sobrepasar el año, como en la bien documentada instancia en que se prolongó durante 34 meses (11). Bryceson afirma que puede ser hasta de 9 años (10). De haberse infectado la niña en San Nicolás, el período de incubación de su leishmaniasis visceral fue de unos 6 meses. Además, la leishmaniasis visceral se presenta en un espectro que va desde infección sin enfermedad, granulomas viscerales asintomáticos, enfermedad oportunista o de reactivación, como se ha visto en algunos pacientes con SIDA (12), hasta enfermedad clínica franca y manifiesta.

\section{Conclusiones}

Nos pareció necesario hacer las consideraciones anteriores por la importancia que tiene establecer la localidad precisa de ciertas enfermedades transmisibles, pues, así se contribuye a conocer mejor la patología geográfica de Colombia, especialmente cuando esta patología se presenta o se adquiere en nichos ecológicos definidos, como ocurre con la leishmaniasis visceral.

\section{Referencias}

1. Gast Galvis A, Rengifo S. Leishmaniasis visceral. Estudio del primer caso diagnosticado en Colombia. Anales Soc Biol (Bogotá) 1944;1(4):161.

2. Corredor A, Ronderos M, Rey M. Leihsmaniasis visceral americana. Boletín epidemiológico nacional (Ministerio de Salud, Bogotá) 1980;6(364):19.

3. Travi BL, Vélez ID, Brutus L, et al. Lutzomyia evansi, an alternate vector of Leishmania chagasi in a Colombian focus of visceral leishmaniasis. Trans $R$ Soc Trop Med Hyg 1990;84:676-7.

4. Instituto Geográfico Agustín Codazzi. Memoria explicativa sobre el mapa ecológico. Zonas de vida o formaciones vegetales de Colombia.Bogotá: Subdirección agrológica, 1977;13(11):1-238.

5. Sherlock IA. Sobre algunas Phlebotomus e Brumptomyia da Colombia (Dipteria, Psychodidae). Mem Inst O Cruz 1962;60(3):321.

6. Gómez Vargas, A. Comunicación preliminar sobre dos casos de leishmaniasis visceral. Rev Fac Med (Bogotá) 1960;28:43.

7. Gómez Vargas, A. Leishmaniasis visceral en Colombia. Presentación de tres casos. Ant Med 1965;15(5):323 (Abstr).

8. Casas M, Angulo VM, Fajardo E. Kala-Azar en Colombia. Act Med Colomb 1983;8(6):301.

9. Chatterjee KD. Parasitology. Protozoology and helminthology in relationship to clinical medicine. Second edition. Calcuta: Ed. Sree Saraswaty Press Ltda., 1959.

10. Bryceson ADM. Visceral leishmaniasis. En: Medical microbiology and infectious diseases. A.I. Braude, editor. W. B. Saunders, Co., 1981:1137.

11. Stone HH, Tool CD, Pugsley WS. Kala-Azar (visceral leishmaniasis). Report of a case with 34-month incubation period and positive Doan-Wright test. Ann Intern Med 1952:36:686.

12. Altés J, Salas A, Riera M, et al. Visceral leishmaniasis: another HIV-associate opportunistic infection? Report of eight cases and review of the literature. AIDS 1991;5: 201-7. 